\title{
Multitarget survey on the Finance Police personnel: assessment of the health condition
}

\author{
Giancarlo Barraco, MD, DDS 1 \\ Stefano Pagano, DDS ${ }^{1}$ \\ Grazia Lupoli, DDS ${ }^{1}$ \\ Alessandro Dolci, DDS 2 \\ Beniamino Colagrosso, MD ${ }^{3}$ \\ 1 Surgical and Biomedical Sciences Department, Uni- \\ versity of Perugia, Italy \\ 2 University of Tor Vergata, Rome, Italy \\ ${ }^{3}$ Operative Health Unit of the General Command of \\ the Finance Police, Rome, Italy
}

Corresponding author:

Giancarlo Barraco, MD, DDS

Surgical and Biomedical Sciences Department, University of Perugia, Italy

Phone: +39075 5853516

Fax: +39 0755855809

E-mail: giancarlo.barraco@unipg.it

\section{Summary}

Introduction. Over the past $\mathbf{1 0 - 1 5}$ years, Italy has undergone a social transformation, and the class of employees and workers has become more economically stable with a higher buying power. Along with the increased expectations of patients on the quality of life, it has now become a priority to make health and social services ready to face users bearing new requirements and different needs.

Objectives. To provide a description of the state of health of the operating personnel of the $\mathrm{Fi}$ nance Police (Guardia di Finanza), including elements for planning the most appropriate interventions for health promotion and prevention.

Methods. The study analyzed the health condition of a group of soldiers (178 subjects, divided into different age classes) by evaluating the effectiveness of a training and information program and subsequently the level of benefit.

Results. The study population showed a good state of health correlated to the quality of life. Although the population voluntarily submitted to health assessment, the rigour of the calls and briefings carried out in the military health unit and the attention of the group to follow instructions on prevention underlined a positive trend, even in behaviours considered as health-risky. Conclusions. Socio-cultural components and the work environment influence the quality of life. In the case of military health care, the specific military organization was useful to monitor the health condition of the population, maximizing the effectiveness of services, enhancing the information and carrying out prevention strategies and demand of care, which should be an example for the public health services.

Key words: general health, prevention programme, life quality, questionnaire on health.

\section{Introduction}

In the past 10-15 years Italy has changed from being a nation with an entrepreneurial middle-class. The employee and working class has become economically more stable and with a higher buying power. Upon this transformation the health condition of this social class could be defined as "tailor made" by describing its demand of health care (1-3). In this respect, together with increased expectations of patients and life quality, it became a priority to prepare the socialheath services to cope with new demands and different needs. The scientific literature and the epidemiological analyses have shown that, on the whole, a middle-high socio-economic and cultural level, in association with an increase of high level specialized performances, determines a better preservation of the health condition compared to subjects with low socioeconomic and cultural level $(2,4,5)$.

This work, to be considered as preliminary, provides a description of the health condition of a population sample yielding indications for planning the most appropriate interventions in health promotion and prevention.

\section{Methods}

The General Command of The Finance Police, medical district service, and a team of the University of Perugia and the University of Tor Vergata, Rome, have carried out a project $(2009-2012)$ to promote health improvement of the operational personnel of the Finance Police through the adoption of social and health protocols and the prevention activity of education and information by the health operators in charge of the control of the afore mentioned personnel.

The subjects took part to the project on a voluntary base, filled an initial self-evaluation questionnaire of their health status, and received information on epidemiology and health hygiene concerning the operational protocol of the study $(6,7)$. 
Thereafter, all the subjects underwent a medical visit including anamnesis data with a digital record (record of the general health profile) that was integrated with data coming from medical charts, radiodiagnostic analyses and analyses provided by the subjects themselves.

Following the analyses to provide and define a set of indicators for the construction of the health profile of the population to be examined, the subjects of the study were reduced to 178 (eliminating those subjects presenting gaps or incorrect data) $(8,9)$.

This final sample underwent follow-up actions and the final compilation of the self-evaluation questionnaire.

Once the study sample was defined, the remaining Italian population was considered as control reference (data obtained by ISTAT and epidemiological sources), assuming that the socio-economic conditions of the Italian citizens, and therefore the risk factors, were similar among them, but different from those of the study sample $(10,11)$.

\section{Results}

The comparison between the national data and those of the study sample showed a remarkable divergence for all the general health indicators.

For example, the oncological assessments concerning the local plans of screening estimating the incidence and prevalence of oncological diseases strongly diverged from those of the analysed group. In fact, in the sampled population there was only one subject with oncological pathology (specifically, prostate cancer).

The 178 subjects had a biased gender distribution with a female proportion of $6 \%$ and a male proportion of $94 \%$; the percent distribution in the work environment was considerably below the national mean (ranging between 10 and $16 \%$ ). The lowest value recorded in the national data is the total absence of women (as among ambassadors, the Court of Accounts and the general power of attorney), while in the public administration as well as in the legal system the range lies between very low levels around $7.4 \%$ and a value that is higher in politics (16\%) and in between at University level. Within public health, among medical doctors of a complex structure the proportion of women is $10 \%$ (head physician women were $6.1 \%$ in 1993).

The study population was mature, with an average age of 46 in men and 60 in women.

Although the population was homogeneous concerning work and life environment, it was heterogeneous concerning geographic origin: $5 \%$ came from Northern Italy, 54\% from Central Italy, 36\% from Southern Italy, and 5\% from abroad.

Sixty percent of women came from Central Italy and $40 \%$ from Southern Italy.

Ninety nine percent of the subjects declared a good general health condition, and an accurate analysis of hospital admissions and therapies for ongoing pathologies actually highlighted a very low proportion compared to the national mean, underlining that the group showed a perception of excellent health conditions, as it actually was (Fig. 1) $(6,7,9)$.

Most subjects were admitted to hospital only once. Therefore, the analysed group would ensure a remarkable working continuity as only $1 / 3$ of the subjects have been admitted to hospitals more than once (Figs. 2, 3). From the available data (unfortunately the results are not reliable) it was not possible to identify whether the new admission to hospital was due to the same pathology so to correlate it to a possible deficit of the health service of reference.

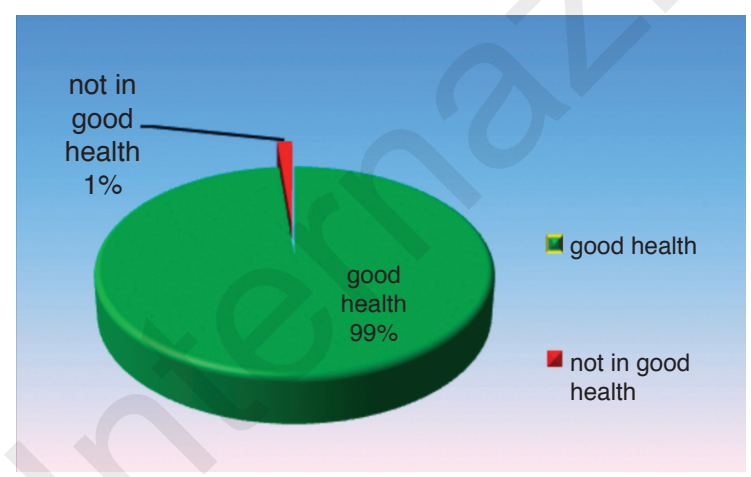

Figure 1. Perception of the health condition of the study population.

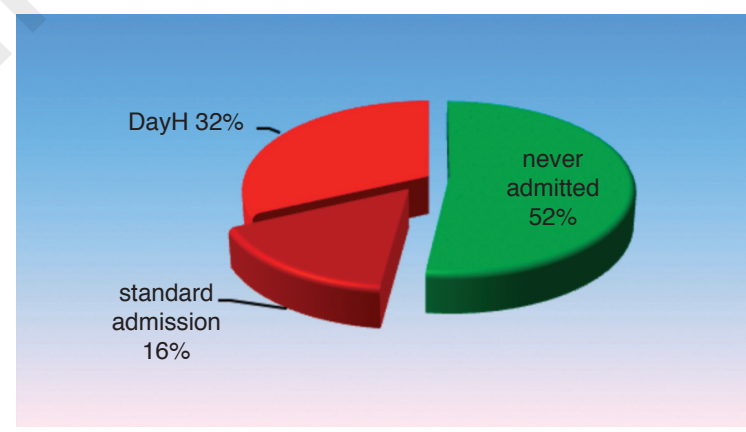

Figure 2. Percent of subjects with standard admissions and day hospital.

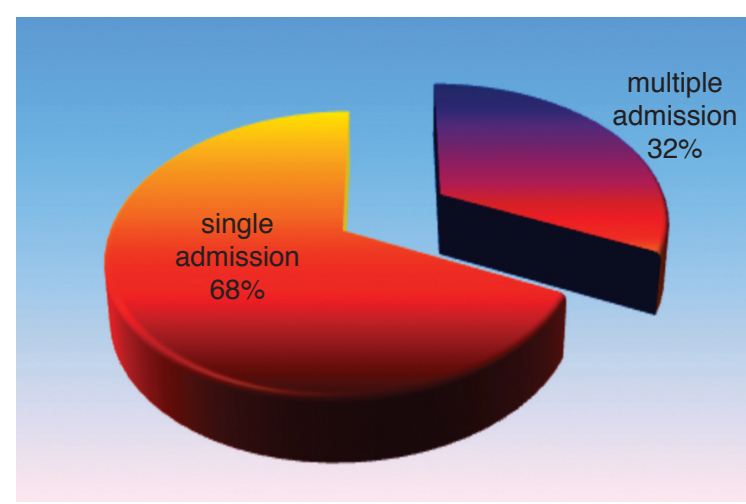

Figure 3. Percent of single and multiple admissions to hospital. 
The causes of admission to hospital were analysed according to the ICD9-CM.

The most common causes of standard admission to hospital in men were represented by traumatic events $(21 \%)$, for which the most frequent age classes involved were between 20 and 40, followed by diseases of the digestive and respiratory trait for which the most frequent age classes were those between 50 and 60.

Day hospital admissions were instead referred to interventions of rhinoplasty followed by hernia and varicocele. For women the comparison with the Italian population showed the highest proportion of hospitalizations due to pathologies correlated to pregnancy, parturition and postpartum, followed by diseases of the urogenital and digestive system, while for men the most common cause of admission to hospital were cardiovascular diseases followed by diseases of the digestive system.

We analysed the distribution of the pathologies. Since the prevalence of hospital admissions was due to traumatic events, we assessed if there was a correlation with traumas associated to place and type of work.

Most traumas were due to car and sport accidents, the latter only involving men of young age classes (Fig. 4). It must be however noticed that a very low number of work traumas occurred (two in total).

In relation to the aetiology of admissions for traumatic events the project of the present work had purposefully preventive aims including evaluation and identification of risky behaviours in order to reduce the social and health costs represented especially by the numerous cases of day hospital.

An interesting result regards the prevalence of lung diseases with asthmatic-allergic manifestations, especially those involving dust (Fig. 5), that diverged from the national mean and could be attributed to a not ideal work environment, such as archives, dusty environments, warehouses, unhealthy rooms with inadequate air turnover, represented by those places where for work reasons (investigations, inspections) the personnel of the Finance Police must carry out his duties; moreover, the increase with age of respiratory chronic pathologies is exponential, not linear, and this strongly supports this hypothesis (12).

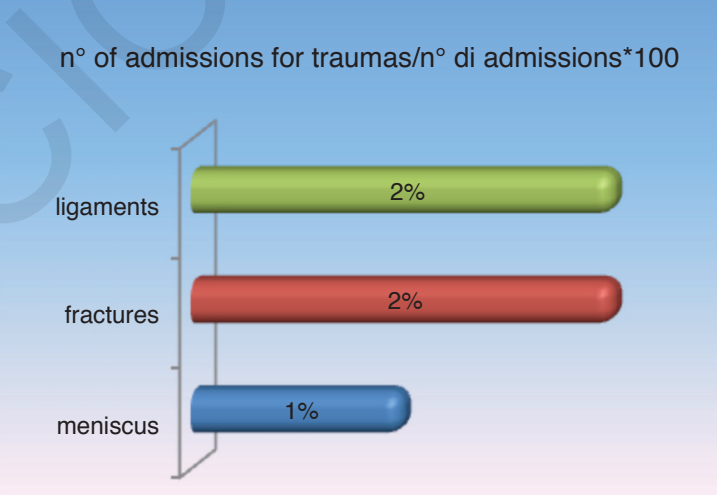

Figure 4. Prevalence of traumatic events on lower limbs.

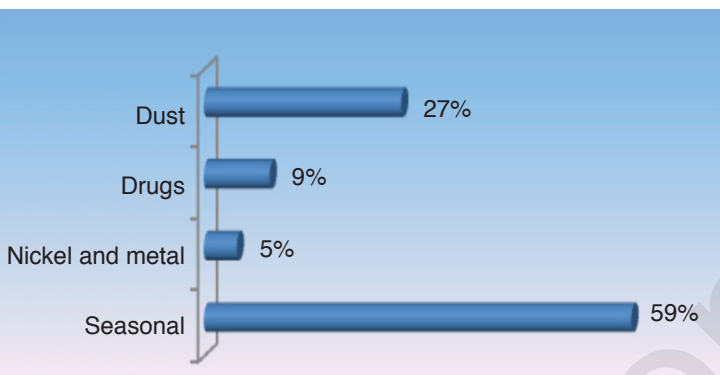

Figure 5. Prevalence of allergic sensitizations.

It must be pinpointed that initially the prevalence of lung pathologies was attributed to pathologies linked to smoking habits, but indeed the prevalence highlights cases specifically correlated to allergic and allergic-asthmatic pathologies, with subsequent BPCO (bronco pneumonitis obstructive pathology).

There were a proportion of subjects with dust allergy, which significantly diverged from the national baseline. The analyses of the respiratory pathologies highlighted that in the study population bronchial asthma constituted about one third of the pathologies, while dust allergies the remaining $2 / 3$.

\section{Discussion}

The aim of this work was to analyse a population from the health point of view, evaluating its health condition.

The goal was to adequately predispose interventions of health education and prevention in an age class that could take advantage of such preventive programs, modifying where possible risky behaviours and lifestyles.

The adequate assistance within the military district under investigation towards patients with respiratory diseases, mediated by careful prevention and health and hygiene education has certainly determined the natural course of diseases, showing that a good coordination among general and specialized medicine allows improving the general profile of the patient and long-term prognosis.

It is therefore evident that in the field of prevention it would be useful to monitor the work environments to reduce at most the possible presence of contaminants with a particular attention to the reduction of risky behaviours and conditions.

However, owing to the nature of the respiratory pathologies emerging from the results, among the objectives at the health military level, it was started a programme of prevention and information with interventions aimed at contrasting the smoking habits as pathogen cofactor, also in relation to the fact that $23 \%$ of the subjects analysed were smokers (against $22.7 \%$ in 2011 and $20.8 \%$ in 2012 at national level) (13-16).

The number of smokers, showing a higher proportion than in the Italian population, is probably due to 
a work situation characterized by stress correlated to the type of tasks, typically investigative and accounting, and to the often unhealthy environmental conditions during inquiries, and this could justify the contrast with the acquisitions emerging from the literature. Indeed, as highlighted by research carried out in Italy before 2003, the "employee" was the high smoking category. Currently, the strict smoking ban in work environments has actively contrasted the working habit at workplaces with positive consequences not only on the health and welfare of the workers, but also on the company budgets; however, for an investigative company as the Finance Police the actual work place is the place where the violation with which the investigation is dealing, not the office.

The other worrying result, strongly diverging from the national mean and other administrative public environments, is the number of gastric and duodenal pathologies that were crossed with more generic data in order to verify the stress condition of the study population with evaluations on incorrect habits, onycophagy, insomnia, anxiety, and other behaviours with an important mental load on the subjects.

For obvious reasons, we can't conclude that the causes originating the psychophysical discomfort underlined by the indicators should be attributed to the work conditions, since there could be environmental confounds and individual personality differences in resistance. However, we believe that such preliminary data deserve to be confirmed with a deeper investigation especially focussed on psychological and stress assessments.

The presence of chronic pathologies, constituting a major indicator to evaluate health condition in terms of worsening of life quality showed a lower frequency of arthrosis-arthritis (the most common disorders in advanced age) compared to the national mean but also of less common diseases with high impact such as diabetes and heart pathologies $(4,6,7)$. In the evaluations we inserted the currently present pathologies and pharmacological therapies. We can conclude that only a minor proportion (10\%) assumed drugs (antihypertensive, antacid, intestinal antispasmodic, oral hypoglycemic, thyroid hormones, anxyolitic).

In the study population $13 \%$ of the subjects reported migraine, differing from most epidemiological studies reporting proportions ranging from $18 \%$ in women and $6 \%$ in men between age 25 and 55 and causing work inability.

It is therefore imperative an early and correct identification of the affected subjects to guarantee an appropriate treatment. Education and updating of the personnel of the health service in recognising and treating on time the forms of migraine are some of the objectives of this prevention project.

Considering the mature age of the study group, the results of the present research show the efficacy of the considerable health control, especially in terms of prevention, obtained thanks to the calls, the periodic analyses, and the adoption of healthy behaviours in the lifestyle (17-19).

\section{Conclusion}

Selecting a homogeneous worker group has the advantage of examining a population with similar needs, common working habits and similar circadian rhythms. In spite of an heterogeneous geographic provenience, an homogeneous working life is commonly present (due o similar food and environmental habits). This study aimed at analysing the health condition and allowed planning a standardization of the therapeutic procedures and prevention with a high cost-benefit ratio.

The improvement of the health condition is facilitated by: rigorous checks and respect of rules that have diminished risky behaviours; efficacy of the military pyramidal structure with well defined roles, competencies, organization, active presence at meetings, and distribution of informative materials; more effective execution of therapeutic and preventive protocols with a consequent improvement of the assistance.

The preliminary assessments already allowed modulating the assistance response by orienting the activities depending on the specific requests and the predictable needs of the workers.

The continuous improvement of the quality of the health assistance with technological instrumental innovations at the frontiers of medicine, the fund raising for the implementation of specialized units, the possibility to offer trained personnel and specific performances with particular attention to the prevention and conformity to healthy lifestyles proved to be points of excellence of the health service offered to the personnel of the Finance Police.

The recommendations of clinical behaviour constituted a fundamental part in the project scheme together with the University team. The identification of "process indicators" and "final result" provided an immediate and evident validation; the individual performances of the health district of the Finance Police in association with the activities of the university team, against the initial information recorded at time zero have shown a validity of the informative activity of the members of the project and was evident in the comparison between the self-evaluation forms.

The recommendations of medical preventive behaviours and those more truly clinical have been checked thanks to the epidemiological evaluations for comparison with the control visits carried out with extreme efficiency by the Head of the Health Service.

\section{References}

1. Materia E, Spadea T, Rossi L, et al. Diseguaglianze nell'assistenza sanitaria: ospedalizzazione e posizione socioeconomica a Roma. Epidemiol Prev. 1999;23:197-201.

2. Vineis $P$, Capri $S$. La salute non è una merce. Efficacia della medicina e politiche sanitarie. Torino, Bollati Boringhieri. 1994.

3. Rapiti E, Perucci CA, Agabiti N, et al. Diseguaglianze socioeconomiche nell'efficacia dei trattamenti sanitari. Tre esempi nel Lazio. Epidemiol Prev. 1999;23:153-160.

4. Vannoni F, Burgio A, Quattrociocchi L, Costa G, Faggiano F. Differenze sociali e indicatori di salute soggettiva, morbosità 
cronica, disabilità e stili di vita nell'Indagine Istat sulla Salute del 1994. Epidemiol Prev. 1999;23:215-229.

5. Kunst E, Cavelaars AEJM, Groenhof F, et al. Socioeconomic inequalities in morbidity and mortality in Europe: a comparative study. Rotterdam, Department of Public Health Erasmus University. 1996.

6. Grant MD, Piotrowski ZH, Chappell R. Self-reported health and survival in the longitudinal study of aging, 1984-86. J Clin Epidemiol. 1995;48:375-387.

7. Hoyemans N, Feskens EJM, Kromhout D, Van Den Bos GAM. Ageing and the relationship between functional status and self-related health in elderly men. Soc Sci Med. 1997;45 (10):1527-1536.

8. Skov T, Deddens J, Petersen MR, Endhal L. Prevalence proportion ratios: estimation and hypothesis testing. Int J Epidemmiol. 1998;27:91-95.

9. Stewart AL, Hays RD, Ware JE jr. The MOS short-form general health survey. Medical Care. 1988;26:724-731.

10. ISTAT: Direzione Centrale delle Statistiche su Popolazione e Territorio. Indagine Multiscopo sulle famiglie. Livello di precisione dei risultati. available on line: http://www.istat.it/it/files/ 2014/06/met_-norme_06_31_il_sistema_di_indagini_multiscopo.pdf. (last access 23-06-2014).

11. ISTAT: Codici dei Comuni, delle Province e delle Regioni. disponibile on line all'indirizzo: http:// http://www.istat.it/it/archivio/6789. (last access 23-06-2014).
12. Scichilone N, Pedone C, Battaglia S, et al. Diagnosis and management of asthma in the elderly. Eur $\mathrm{J}$ Intern Med. 2014;25:336-342.

13. Mc Phillips JB, Eaton CB, Gans KM, et al. Dietary differences in smokers and non smokers from two south eastern New England communities. J Am Diet Assoc 1994;94:287-292.

14. Morabia A, Wynder EL. Dietary abits of smokers, people who never smoked and ex smokers. Am J Clin Nutr. 1990;59: 11365-11425.

15. Office on Smoking and Health: Smoking and Health: A National Status Report, Second Edition. A Report To Congress. available on line: http://profiles.nlm.nih.gov/ps/access/NNBBVP.pdf. (last access 23-06-2014).

16. Townsend J. The burden of smoking. In Benzeval M, Judge K, Whitehead $M$ (eds). Tackling Inequalities in Health. An agenda for action. London, King's Fund Institute. 1995:82-94.

17. Whitehead M. Swimming Upstream: Trends and Prospects in Education for health. Research Report n. 5. London, King's Fund Institute. 1989.

18. WHO: Oral Health Country/Area Profile programme. disponibile on line all'indirizzo: http://www.who.int/oral_health/databases/malmo/en/. (last access 23-06-2014).

19. WHO: Indagini sulla salute orale: metodi di base. Quarta edizione-Traduzione Italiana Istituto di Scienze Biomediche San Paolo, Università degli Studi di Milano. 1997. 